\title{
Position Sensorless Control of Switched Reluctance Motor under Magnetic Field Saturation
}

\author{
Chunyuan Qiu ${ }^{1 *}$, Baojiang Sun ${ }^{1}$ \\ ${ }^{1}$ Qilu University of Technology (Shandong Academy of Sciences), Jinan, Shandong, China
}

\begin{abstract}
When the switched reluctance motor is running, as the motor phase current increases, the magnetic circuit of the motor will gradually saturate, which affects the estimation of the special position of the motor rotor. Aiming at the above problems, this paper proposes a positionless control method for switched reluctance motors under the condition of magnetic field saturation. Firstly, the Fourier phase inductance function of switched reluctance motor is established, and then the component of Fourier phase inductance function affected by magnetic field saturation is eliminated mathematically. Research the method of estimating the position and angle of the rotor based on the inductance positioning point. This paper takes a $12 \backslash 8$ motor as an example to verify the effectiveness of the method by simulation.
\end{abstract}

\section{1 introduction}

Switched reluctance motor (SRM), as a new type of electromechanical integrated speed control system, has the characteristics of simple structure, strong robustness and high reliability.[1-2] This type of motor is widely used in mining, public transportation, textile machinery and many other fields. The key to the ability of a switched reluctance motor to rotate is to detect the position of the rotor and to switch between different phases on and off in time to change the magnetic field of the motor and generate a different electromagnetic force than before the commutation.

At present, there are many kinds of non-position control methods for switched reluctance motors. Most of these methods use the correspondence relationship between flux linkage-current-inductance to estimate the position of the rotor. For example, use the relationship between inductance and current to determine the inductance by testing the current, and then determine whether it should be changed phase by the change of the inductance. [3-4]

Aiming at the saturation of the phase inductance of the $12 \backslash 8$ motor in the case of high current, this paper proposes a method to estimate the rotor position of the motor based on the special position of the inductance.[5]

\section{Switched reluctance motor phase inductance function}

According to the method of determining the rotor position of the switched reluctance motor based on the phase inductance positioning point proposed in this paper, the phase inductance function of each phase needs to be determined. According to the slope method, the inductance expression of the three-phase switched reluctance motor can be obtained as:

$$
\mathrm{L}(\theta)=\frac{2 U_{a}}{\frac{d i}{d t} l_{o n}-\left.\frac{d i}{d t}\right|_{o f f}}=\frac{2 U_{a}}{\Delta i}
$$

In the formula, $\Delta \mathrm{i}$ is the slope difference of the phase current between the MOS tube on and off. According to formula (1), a series of inductance values at different positions and angles in the full cycle of the SRM can be calculated, and the corresponding functional relationship can be obtained by the method of numerical fitting.

\subsection{Establish a Fourier series model}

The Fourier expansion of the inductance can be expressed as equation (2).

$$
\mathrm{L}=\sum_{j=0}^{\infty} L_{j}(i) \cos \left(j N_{r} \theta+\varphi_{j}\right)
$$

In the above formula, $N_{r}$ is the number of rotor poles; $L_{j}(i)$ is the coefficient function of the fundamental wave and each harmonic term; $\varphi_{j}$ is the initial phase. For a $12 / 8$ switched reluctance motor, an electrical cycle is $45^{\circ}$, and it is symmetrical about $22.5^{\circ}$, so this article uses special position points $0^{\circ}\left(L_{a}\right), 7.5^{\circ}\left(L_{b}\right), 11.25^{\circ}\left(L_{c}\right), 15^{\circ}\left(L_{d}\right)$, $22.5^{\circ}\left(L_{e}\right)$ Fourier series expansion coefficient $L_{0}(\mathrm{i}), L_{1}(\mathrm{i})$ and $L_{2}(\mathrm{i})$. Its decomposition formula is formula (3), (4), (5).

$$
\begin{array}{r}
L_{A}\left(\theta_{e}\right)=L_{0}\left(i_{A}\right)+L_{1}\left(i_{A}\right) \cos \left(\theta_{e}+\frac{2 \pi}{3}\right)+ \\
L_{2}\left(i_{A}\right) \cos \left[2\left(\theta_{e}+\frac{2 \pi}{3}\right)\right] \\
L_{B}\left(\theta_{e}\right)=L_{0}\left(i_{B}\right)+L_{1}\left(i_{B}\right) \cos \left(\theta_{e}\right)+L_{2}\left(i_{B}\right) \cos \left(2 \theta_{e}\right) \\
L_{C}\left(\theta_{e}\right)=L_{0}\left(i_{C}\right)+L_{1}\left(i_{C}\right) \cos \left(\theta_{e}-\frac{2 \pi}{3}\right)+ \\
L_{2}\left(i_{C}\right) \cos \left[2\left(\theta_{e}-\frac{2 \pi}{3}\right)\right](5)
\end{array}
$$

Use special position points to express $L_{0}(\mathrm{i}), L_{1}(\mathrm{i})$ and 
$L_{2}(i)$ the mathematical expression is:

$$
\begin{gathered}
L_{0}(i)=\frac{1}{6}\left(L_{a}+L_{e}\right)+\frac{1}{3}\left(L_{b}+L_{d}\right) \\
L_{1}(i)=\frac{1}{3}\left(L_{a}+L_{b}-L_{d}-L_{e}\right) \\
L_{2}(i)=\frac{1}{4}\left(L_{a}-2 L_{c}+L_{e}\right)
\end{gathered}
$$

In the actual mid-phase inductance modeling, the Fourier series formula is used to model, only the fundamental wave or the second harmonic is considered, and the higher harmonics are ignored. It can be seen from the literature [19] that when the Fourier series is used to express the single-phase inductance of the motor, the sum of the three-phase inductance is a constant. However, it can be known through simulation and experiment that the sum of the three-phase inductance has a certain fluctuation phenomenon, which shows that the sum of the three-phase inductance is not an exact constant. This shows that the high-order harmonic inductance of SRM cannot be ignored.

\subsection{Fourier series inductance harmonic analysis}

\subsubsection{Harmonic elimination based on inductance coordinate transformation}

Taking the phase $\mathrm{B}$ inductor as an example, define the position where the concave pole of the motor rotor and the centerline of the salient pole of the motor stator overlap as the zero point. Due to the single-phase conduction excitation of the motor, the mutual inductance effect between the windings can be ignored. The Fourier series expression of phase $\mathrm{B}$ is:

$$
L_{B}=L_{0}+\sum_{n=1}^{\infty}\left[L_{n} \cos (n \omega t)\right]
$$

Delay the inductance of phase B by $\pi$ to obtain:

$$
L_{B d}=L_{0}+\sum_{n=1}^{\infty}\left[L_{n} \cos \mathrm{n}(\omega t-\pi)\right]
$$

Combining formula (9) and formula (10), the difference between the phase $\mathrm{B}$ inductance before and after the delay is obtained:

$$
\Delta L_{B}=L_{B}-L_{B d}
$$

In the same way, $\Delta L_{A}$ and $\Delta L_{C}$ can be obtained. After the coordinate transformation, the coordinate expression of the three-phase inductance is:

$$
\left\{\begin{array}{c}
\Delta L_{A}=2 \sum_{n=1}^{\infty}\left[L_{n} \cos n\left(\omega t-\frac{2 \pi}{3}\right)\right] \\
\Delta L_{B}=2 \sum_{n=1}^{\infty}\left[L_{n} \cos n(\omega t)\right] \\
\Delta L_{C}=2 \sum_{n=1}^{\infty}\left[L_{n} \cos n\left(\omega t+\frac{2 \pi}{3}\right)\right] \\
(n=2 m+1, m \geq 0)
\end{array}\right.
$$

It can be seen from the above formula that the inductor $\Delta L_{B}$ is in phase with the inductor $L_{B}$, and the DC component and even harmonic components of $\Delta L_{B}$ are eliminated.

\subsubsection{Eliminate harmonic inductance by making difference}

In order to further eliminate the harmonic components of the inductor, on the basis of the above, make the difference of $\Delta L_{A}, \Delta L_{B}$ and $\Delta L_{C}$ to obtain $\Delta L_{A B}, \Delta L_{B C}$ and $\Delta L_{C A}$. Because the three-phase inductance of the switched reluctance motor is relatively static in the A-B-C coordinates and differs from each other by $120^{\circ}$. Therefore,
$\Delta L_{A B}, \Delta L_{B C}$ and $\Delta L_{C A}$ are also different from each other $120^{\circ}$, and the magnitude changes with the inductance of the three phases A, B, and C. It can be seen from the symmetry of the three-phase inductance:

$$
\left\{\begin{array}{l}
\Delta L_{A B}=\Delta L_{A}-\Delta L_{B} \\
\Delta L_{B C}=\Delta L_{B}-\Delta L_{C} \\
\Delta L_{C A}=\Delta L_{C}-\Delta L_{A}
\end{array}\right.
$$

Bring $\Delta L_{A}, \Delta L_{B}$ and $\Delta L_{C}$ into equation (21) to equation (22).

$$
\left\{\begin{array}{c}
\Delta L_{A B}=\sqrt{3} \sum_{n=1}^{\infty} L_{n}\left[\cos \left(n \omega t\left(\cos \frac{2 n \pi}{3}-1\right)\right)+\sin (n \omega t) \sin \frac{2 n \pi}{3}\right] \\
\Delta L_{B C}=\sqrt{3} \sum_{n=1}^{\infty} L_{n}\left[\cos \left(n \omega t\left(1-\cos \frac{2 n \pi}{3}\right)\right)+\sin (n \omega t) \sin \frac{2 n \pi}{3}\right] \\
\Delta L_{C A}=-\sqrt{3} \sum_{n=1}^{\infty} L_{n}\left[\sin (n \omega t) \sin \frac{2 n \pi}{3}\right]
\end{array}\right.
$$

$$
(n=2 m+1, m \geq 0)
$$

Through formula (14), it can be found that the three times harmonic component of the inductance of the Fourier series is eliminated.

\section{Rotor speed and position angle estimation}

\subsection{Determination of rotor speed}

After the above coordinate transformation and the change of the difference of the inductance, the DC component of the inductance, the even harmonic component and the harmonic component of multiples of three have been completely eliminated, and at the same time, other harmonic components have become very small. Based on the above analysis results, this article will replace $L_{A}, L_{B}$ and $L_{C}$ with $\Delta L_{A B}, \Delta L_{B C}$ and $\Delta L_{C A}$ as the three-phase inductance for estimating the rotor position. By detecting the intersection between two adjacent difference inductances, the rotor speed can be estimated. P1, P3, and P5 are selected as the feature points of the estimated position. Transmit position pulses at the characteristic points to estimate the position and angle of the motor rotor When the logical relationship corresponding to the difference inductance is detected, a pulse signal is transmitted. The logical relationship is shown in Table 1.

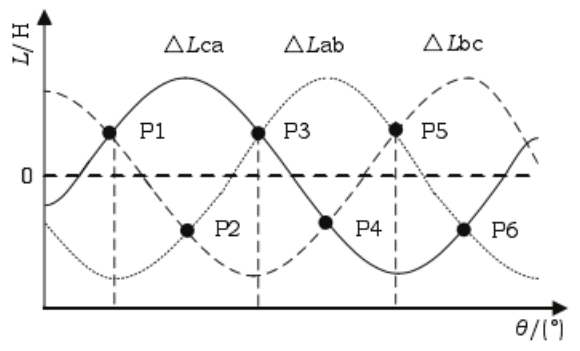

Figure 1 Schematic diagram of the intersection of differential inductance curves

Table 1 Logical relationship table of difference inductance

\begin{tabular}{|c|c|}
\hline Logic & Feature points \\
\hline$\Delta \boldsymbol{L}_{\boldsymbol{B C}}=\Delta \boldsymbol{L}_{\boldsymbol{C A}}>\Delta \boldsymbol{L}_{\boldsymbol{A B}}$ & P1 \\
\hline$\Delta \boldsymbol{L}_{\boldsymbol{C} \boldsymbol{A}}=\Delta \boldsymbol{L}_{\boldsymbol{A B}}>\Delta \boldsymbol{L}_{\boldsymbol{B C}}$ & P3 \\
\hline$\Delta \boldsymbol{L}_{\boldsymbol{A B}}=\Delta \boldsymbol{L}_{\boldsymbol{B} \boldsymbol{C}}>\Delta \boldsymbol{L}_{\boldsymbol{C A}}$ & P5 \\
\hline
\end{tabular}


According to the above three characteristic points $\mathrm{P} 1$, $\mathrm{P} 3$, and $\mathrm{P} 5$, a $45^{\circ}$ inductance periodic waveform is divided into three parts, and each part occupies $15^{\circ}$. Count the speeds of these three intervals, and take the average of these speeds as the average rotor speed of the inductance period. The average rotation speed between two characteristic location points is:

$$
\omega=\frac{\Delta \theta}{\Delta t}
$$

In formula (15), $\omega$ is the average speed of the motor between two characteristic positions; $\Delta \theta$ is the rotor displacement angle between the two characteristic positions; $\Delta \mathrm{t}$ is the time between the position pulses of the two special positions.

\subsection{Determination of rotor position and initial characteristic points}

Knowing the motor rotor speed, in order to obtain the final motor rotor angle, an initial angle of the rotor position in a known interval is required. We take $\mathrm{P} 1$ as the initial position feature point, and find the initial position of $\mathrm{P} 1$. Let $\Delta \boldsymbol{L}_{\boldsymbol{B} \boldsymbol{C}}=\boldsymbol{\Delta} \boldsymbol{L}_{\boldsymbol{C} \boldsymbol{A}}$, the following formula can be obtained, and the first characteristic location point P1 can be obtained.

$$
\frac{\cos \left(\theta-\frac{2 \pi}{3}\right)+\cos \theta-2 \cos \left(\theta+\frac{2 \pi}{3}\right)}{2 \cos \left(\theta+\frac{2 \pi}{3}\right)-\cos 2 \theta-\cos 2\left(\theta-\frac{2 \pi}{3}\right)}=\frac{L_{2}(i)}{L_{1}(i)}
$$

In the same way, the special position points $\mathrm{P} 2$ and $\mathrm{P} 3$ can also be obtained by the above-mentioned method, namely:

$$
\begin{gathered}
\Delta L_{C A}=\Delta L_{A B} \\
\Delta L_{A B}=\Delta L_{B C}
\end{gathered}
$$

Finally, use the difference inductance logic table in Table 1 to determine the specific location point. The initial position point and the average speed of the motor can be used to determine the position angle of the SRM rotor. It can be obtained by the following formula:

$$
\theta(\mathrm{t})=\theta_{\mathrm{p}}\left(t_{p}\right)+\omega\left(t-t_{p}\right)
$$
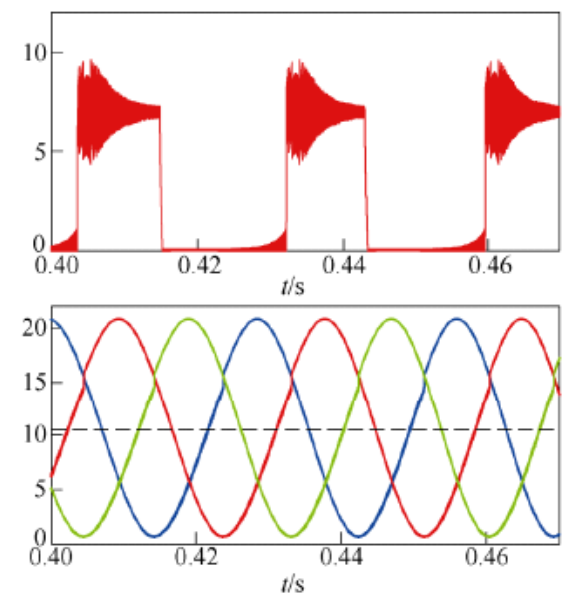

(a)Phase inductance and phase current

\section{Simulation analysis}

In order to verify the correctness of the above algorithm, this article builds a 12/8 switched reluctance motor model in the MATLAB $\backslash$ Simulink environment and conducts simulation experiments. The inner loop adopts current chopping control mode. The outer loop adopts PI control. The calculation of the rotor position of the motor is first brought into the phase inductance calculation formula through the phase voltage to obtain the inductance value of each phase, and then calculate $\Delta L_{A B}, \Delta L_{B C}, \Delta L_{C A}$ to determine whether the current inductance value is the inductance value at the positioning point, and then Determine the position angle and speed of the SRM rotor through the positioning of the phase inductance. Above and below the critical saturation current of 9A, any switched reluctance motor phase A currents of 7A, 10A, and $16 \mathrm{~A}$ are used for simulation verification at a speed of $600 \mathrm{r} / \mathrm{min}$. The corresponding simulation waveform is shown below.

However, as the current increases, it can be seen from Figure 2, Figure 3, Figure 4 that the inductance waveform gradually deforms. Under the premise of greater than the critical current of 9A, it can be seen from Fig. 3 and Fig. 4 that using the algorithm in this article, the detection of special position points is not much different from that when the current is 7A. Table 3 shows the maximum deviation between the position estimated by the algorithm and the actual rotor position of the motor under different currents. Therefore, the algorithm proposed in the article can effectively estimate the position of the motor rotor, and the maximum angle error does not exceed 0.9 .

Table 3 Maximum angle error table

\begin{tabular}{|c|c|}
\hline Current/A & Maximum angle error \\
\hline 7 & 0.8 \\
\hline 10 & 0.9 \\
\hline 16 & 0.9 \\
\hline
\end{tabular}
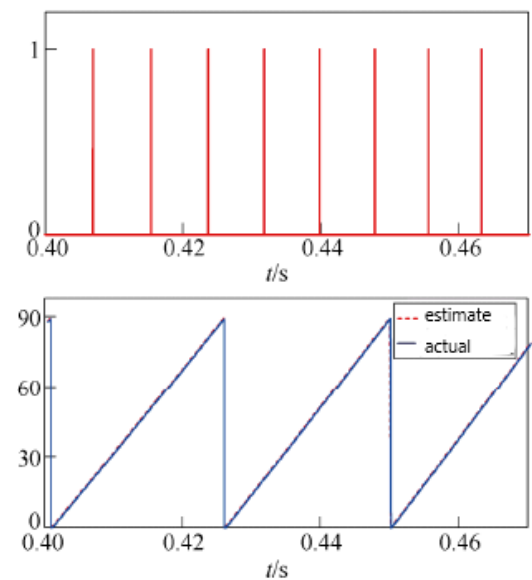

(b) Intersection signal and rotor position signal

Figure 2 Simulation waveform when the current is 7A 

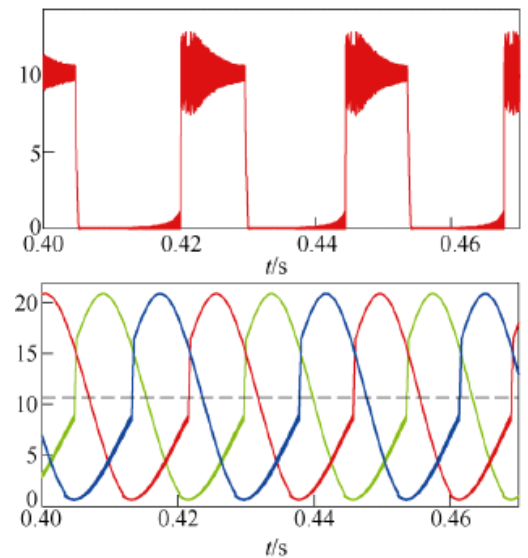

(a) Phase inductance and phase current
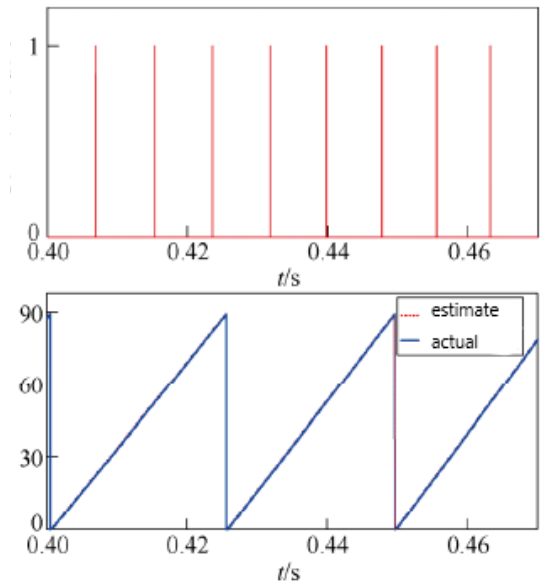

(b) Intersection signal and rotor position signal

Figure 3 Simulation waveform when the current is $10 \mathrm{~A}$
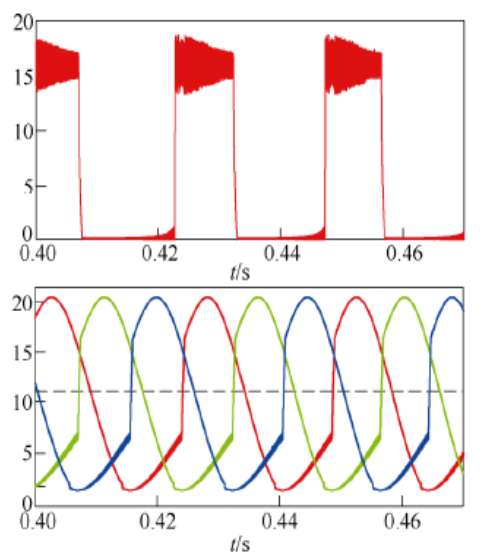

(a) Phase inductance and phase current
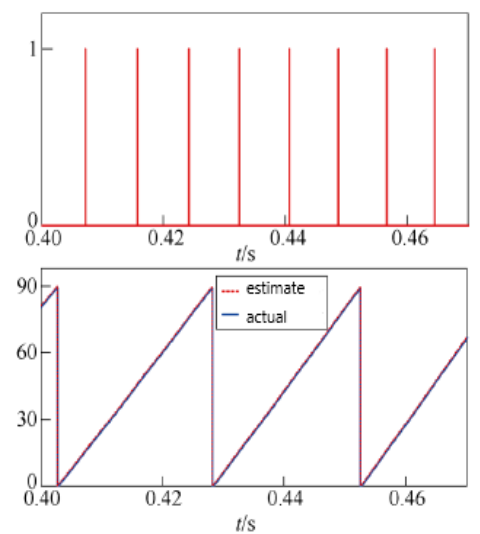

(b)I ntersection signal and rotor position signal

Figure 4 Simulation waveform when the current is $16 \mathrm{~A}$

\section{5 conclusion}

Aiming at the inaccurate estimation of the rotor position angle of the motor due to the saturation of the magnetic circuit caused by the increase of current in the operation of the switched reluctance motor, a Fourier series using inductance is proposed to remove excessive magnetization. The simulation was carried out in the MATLAB $\backslash$ Simulink environment to verify the correctness of the algorithm in this paper.

\section{References}

1. Bhuvaneswari S, Sivaraman $\mathrm{P}$, Maheswari K T,Matheswaran A. (2021)Super Core Magnetic Material based Switched Reluctance Motor for Electric Vehicle Applications[J]. IOP Conference Series: Materials Science and Engineering, 1084(814).

2. Huang J L,Xuan Y, Zhang L, Liu T G.(2021) Analysis on the design and temperature field of switched reluctance motor for electric vehicle[J]. Journal of Physics: Conference Series, 1777(9-15).

3. Fantin Irudaya Raj E., Balaji M..(2020) Analysis and Classification of Faults in Switched Reluctance
Motors Using Deep Learning Neural Networks[J]. Arabian Journal for Science and Engineering,46(2-8)

4. Rajendran A.,Karthik B..(2020) Design and analysis of fuzzy and PI controllers for switched reluctance motor drive[J]. Materials Today: Proceedings, (prepublish).

5. Megha D. Chaple, Sanjay B. (2020) Bodkhe. An integrated converter model and enhanced control approach for torque ripple minimisation of switched reluctance motor: a CMFG-RNN technique[J]. International Journal of Ambient Energy,41(10-16). 